\title{
Specific patient challenge: The elderly hypertensive patient
}

MICHAEL L. TUCK, M.D. Sepulveda, California
In a multicenter study of low-dose captopril, 97 elderly hypertensive patients were treated initially with captopril monotherapy (25 mg. twice daily). Nonresponders were assigned to treatment with either a doubled dose of captopril or a twicedaily combination of $25 \mathrm{mg}$. of captopril and $15 \mathrm{mg}$. of hydrochlorothiazide. Overall, 74 (76 percent) of the patients studied demonstrated a satisfactory response to therapy. Black and white patients responded similarly. Among patients who remained uncontrolled with the initial dosage, the group receiving the combination therapy showed a greater improvement than those assigned to $50 \mathrm{mg}$. of captopril twice daily. Only 5 percent of patients were discontinued because of side effects, and no biochemical alterations, episodes of orthostatic hypotension, or deterioration of renal function occurred. Captopril appears to be a highly suitable antihypertensive drug for use in elderly patients, including those with concomitant diseases such as diabetes, heart failure, chronic pulmonary disease, and peripheral vascular disease. In addition, its relative freedom from central side effects and adverse hemodynamic changes should enhance the quality of life for geriatric patients who require antihypertensive therapy.

Despite the high prevalence of hypertension among elderly patients, their treatment for this disease has not been optimal. In fact, one of the more persistent medical notions has been that older individuals are, for some reason, spared the cardiovascular and other adverse effects of high blood pressure and that treatment of their hypertension, therefore, should not be a high priority. This prevailing thought was countered by the results of the Framingham Study, ${ }^{1}$ which showed a gradient risk in every decade up to age 80 for stroke, congestive heart failure, and coronary artery disease.

The beneficial effect of therapy for hypertension in the older patient has been demonstrated only recently. Both the Veterans Administration Cooperative Study ${ }^{2}$ and the Hypertension Detection and Follow-up Program ${ }^{3}$ provided preliminary evidence of a reduction in mortality among effectively treated older hypertensive patients. The European Working Party on High Blood Pressure in the Elderly (EWPHE) study ${ }^{4}$ included 840 hypertensive patients older than 60 years of age who were randomized to treatment with either placebo $(424 \mathrm{pa}-$ tients) or a combination of hydrochlorothiazide and a potassium-sparing diuretic (416 patients). In the absence of a satisfactory response, the dosage of active drug could be doubled, and a second-step agent (methyldopa) could be added if blood pressure remained uncontrolled. With treatment, mean reductions of approximately $32 \mathrm{~mm}$. $\mathrm{Hg}$ systolic blood pressure and approximately $13 \mathrm{~mm}$. Hg diastolic pressure were observed; these reductions were maintained over a 1- to 5-year course of evaluation. Results of the EWPHE study showed that its incidences of cardiac mortality and congestive heart failure were decreased by 38 percent and 63 percent, respectively, in the active treatment group. Thus, the EWPHE study provides definitive evidence that effective drug therapy can reduce morbidity and mortality among elderly hypertensive patients.

Selection of drug therapy for the elderly hypertensive patient is not always straightforward, as many factors make this population special as compared to younger hypertensive patients. Hypertension in the older patient is characterized by high peripheral resistance, low cardiac output, low plasma volume, and reductions in both baroreceptor and beta-adrenoreceptor sensitivities. ${ }^{5,6}$ Also, renal function declines with age, and older patients do not handle the extremes of salt and water perturbations as well as younger individuals. Because of their impaired renal concentrating capacity, elderly patients lose salt and water very readily under conditions of dehydration. ${ }^{5}$ Conversely, when 
volume expands, they have difficulty in excreting salt. In addition, older patients exhibit some major changes in hormonal systems that regulate blood pressure. Activity of the renin-angiotensin system declines, while sympathetic nervous system activity, as estimated by plasma levels of norepinephrine, appears to increase with advancing age.

Another consideration in the treatment of hypertension in elderly persons is the existence of concurrent diseases. The significant incidences of diabetes mellitus, degenerative joint disease, congestive heart failure, and angina in this population make it important that one selects antihypertensive medications that do not worsen the deleterious processes associated with these conditions and that do not interact adversely with concomitantly prescribed medications.

Finally, one must consider the differences in pharmacokinetics of medications when they are administered to elderly individuals, as well as the observation that older patients may be more sensitive than the general population to the adverse effects of antihypertensive drugs. ${ }^{7}$ For example, the Boston Collaborative Drug Surveillance Study showed an association between elevated levels of propranolol and an increased level of adverse effects in this population. ${ }^{8}$ In reviewing the agents most frequently used for the treatment of hypertension during the past decade, it is apparent that while each has associated benefits, none are free of disadvantages for the older patient. The metabolic effects of diuretics, the antihypertensive drugs used most commonly in elderly patients, have been a matter of discussion for some time, and there are good reasons to believe that these complications may be accentuated in an older population. For example, in the EWPHE trial, ${ }^{4}$ mean increases in serum creatinine, serum uric acid, and fasting blood glucose values were observed in the active treatment group. The fall in serum potassium was small in this study, because the treated patients were receiving a potassium-sparing diuretic. While the relative importance of these biochemical effects as compared to other risk factors is still not clear, they remain an area of concern and suggest that alternative antihypertensive therapies may be best for the elderly population.

Some of the advantages of beta-blockers for older patients include their antianginal effects and the possibility that they may be cardioprotective, although most evidence would suggest that the secondary cardioprotection phenomenon may not extend past age $65 .{ }^{9}$ Disadvantages of these agents for elderly persons are their central side effects and their tendency to reduce cardiac output, when these patients already have diminished output. In addition, it has been suggested that the efficacy of beta-blocker monotherapy in elderly hypertensive patients is reduced as compared to younger hypertensive subjects. ${ }^{10}$

Centrally acting agents such as methyldopa and clonidine are effective against geriatric hypertension and do not compromise cerebral or renal function, but their common side effects of sedation, dry mouth, and orthostatic hypotension may not be well tolerated by the elderly patient. The central nervous system side effects can limit the quality of life in frail, older patients, and the orthostatic hypotension encounted with methyldopa and prazosin is a potentially serious adverse event.

The angiotensin-converting enzyme inhibitor captopril has a hemodynamic profile that may be suitable for the elderly patient, as it produces peripheral vasodilatation without reflex tachycardia. Because of its specific pharmacologic activity, captopril preserves cerebral and renal blood flow as well as baroreceptor sensitivity. Although elderly patients usually have a low-renin form of hypertension, captopril is effective through mechanisms not related to renin status. ${ }^{11}$ In order to assess the advantages of captopril in older patients, a study of low doses of captopril was conducted in the geriatric population.

\section{Patients and methods}

This multicenter study enrolled men and women older than 60 years with mild-to-moderate hypertension and normal renal function. ${ }^{12}$ Informed consent was obtained from each participant. After a 2week placebo lead-in period, patients were started on captopril at a dosage of $25 \mathrm{mg}$. twice daily. Upon completing 2 weeks of active drug therapy, responders were maintained on the initial dosage of captopril, while patients with a diastolic blood pressure that remained above $90 \mathrm{~mm}$. $\mathrm{Hg}$ were assigned randomly to either captopril, $50 \mathrm{mg}$. twice daily, or a twice-daily combination of $25 \mathrm{mg}$. of captopril and $15 \mathrm{mg}$. of hydrochlorothiazide.

\section{Results}

A total of 97 patients with a mean age of $65.7 \pm 5.4$ years completed the placebo treatment period and were treated with active medication. Blood pressure fell from a mean value of $159 / 99 \mathrm{~mm}$. $\mathrm{Hg}$ at entry to $142 / 87 \mathrm{~mm} . \mathrm{Hg}(p<0.05)$ by the end of the study. Overall, 74 patients ( 76 percent) demonstrated a favorable response to therapy (a reduction in diastolic pressure to $\leqslant 90 \mathrm{~mm}$. $\mathrm{Hg}$, or a $10-\mathrm{mm}$. $\mathrm{Hg}$ reduction from baseline). Black $(\mathrm{n}=41)$ and white $(n=56)$ patients responded equally well to treatment. The average blood pressure drop in 
black patients was $-9.5 /-12.0 \mathrm{~mm}$. $\mathrm{Hg}$, whereas that in white patients was $-11.1 /-13.1 \mathrm{~mm}$. $\mathrm{Hg}$.

Of the patients who were not adequately controlled with the initial dose of captopril, those randomized to treatment with the combination therapy $(n=22)$ experienced greater diastolic blood pressure reductions $(-12 \mathrm{~mm}$. $\mathrm{Hg})$ than those $(\mathrm{n}=23)$ assigned to therapy with double-dose captopril $(-5 \mathrm{~mm}$. $\mathrm{Hg})$. No serious adverse reactions occurred, and only 5 percent of patients were discontinued from the study because of side effects, including rash $(n=1)$, dizziness $(n=1)$, and gastrointestinal upset $(n=1)$. One patient had a paradoxic increase in blood pressure and one experienced a "shaky" feeling. There were no episodes of orthostatic hypotension. No significant changes were seen in serum electrolyte values or other blood chemistry determinations, and there were no alterations in urinary protein excretion.

\section{Discussion}

The findings in the present study were in general agreement with the results of other evaluations of captopril in elderly patients. In a large surveillance trial $^{13}$ involving nearly 1,000 patients older than 65 years, blood pressure was well controlled with either captopril alone or captopril plus a diuretic and/ or another antihypertensive agent. In a small study ${ }^{14}$ among geriatric patients that compared captopril with chlorthalidone, the drugs were equally effective. However, significant changes in serum potassium and serum glucose levels were observed in the diuretic-treated group. Also, in a recent evaluation ${ }^{15}$ of therapy with captopril plus hydrochlorothiazide in patients over 65 years of age, the combination was found to be highly effective, and no important side effects or biochemical changes were reported. ${ }^{15}$

The 5 percent incidence of discontinuation for adverse reactions in the present trial was low and similar to that reported in other studies. ${ }^{13,16}$ Previously reported ${ }^{17}$ differences between black and white patients in their response to captopril were not seen in our study. Like elderly patients, blacks usually are considered to have a volumedependent, low-renin type of hypertension, and the favorable effect of captopril in these patients may be due to its concurrent actions on non-renin-dependent mechanisms of lowering blood pressure.

\section{Conclusions}

Low-dose captopril, alone or in combination with low-dose hydrochlorothiazide, provides a beneficial reduction in blood pressure in elderly patients without adverse metabolic or hemodynamic changes or troublesome central nervous system side effects. It is highly suitable for use in patients with common concurrent diseases, including diabetes, heart failure, chronic obstructive pulmonary disease, and peripheral vascular disease. The angiotensin-converting enzyme inhibitor should contribute significantly to better compliance and to an improved quality of life among treated elderly hypertensive patients.

1. Kannel, W.B.: Hypertension, blood lipids, and cigarette smoking as corisk factors for coronary heart disease (Framingham Heart Study). Ann NY Acad Sci 304:128-39, Mar 78

2. Veterans Administration Cooperative Study Group on Antihypertensive Agents. Effects of treatment on morbidity in hypertension. II. Results in patients with diastolic blood pressure averaging 90 through 114 mm./Hg. JAMA 213:1143-50, 17 Aug 70

3. Hypertension Detection and Follow-up Program Cooperative Group: Five-year findings. 1. Reduction in mortality of persons with high blood pressure, including mild hypertension. JAMA 242:2562-71, 7 Dec 79

4. Amery, A., et al.: Mortality and morbidity results from the European Working Party on High Blood Pressure in the Elderly trial. Lancet 1:1349-54, 15 Jun 85

5. Reed, G., and Anderson, R.J.: Physiological effects of aging and pathophysiology of hypertension in the elderly. Clin Ther 5:8-12, 1982

6. Cressman, M.D., Vidt, D.G., and Gifford, R.W.: Geriatric hypertension controversies (part I). Initial Tx. Geriatrics 40:45-60, Sep 85

7. Hale, W.E., Stewart, R.D., and Marks, R.G.: Central nervous system symptoms of elderly subjects using antihypertensive drugs. J Am Geriatr Soc 32:5-10, Jan 84

8. Greenblatt, D.J., and Koch-Weser, J.: Adverse reactions to propranolol in hospitalized medical patients. Am Heart J 86:478-84, Oct 78

9. Yusuf, S., et al.: Beta blockade during and after myocardial infarction. An overview of the randomized trials. Prog Cardiovasc Dis 27:335-71, Mar-Apr 85

10. Bühler, F.R.: Age and cardiovascular response adaptation. Determinants of an antihypertensive treatment concept primarily based on betablockers and calcium entry blockers. Hypertension 5:94-100, Sep-Oct 83 11. Zusman, R.M., et al.: Renin- and non-renin-mediated antihypertensive actions of converting enzyme inhibitors. Kidney Int 25:969-83, 1984 12. Tuck, M.L., et al.: Low-dose captopril in mild to moderate geriatric hypertension. J Am Geriatr Soc 34:693-6, 1986

13. Jenkins, A.C., Knill, J.R., and Dreslinski, G.R.: Captopril in the treatment of the elderly hypertensive patient. Arch Intern Med 145:2029-31, Nov 85

14. Corea, L., et al.: Converting enzyme inhibition vs. diuretic therapy as first therapeutic approach to the elderly hypertensive patient. Curr Ther Res 36:347-51, Aug 84

15. Creisson, C., Baulac, L., and Lenfant, B.: Captopril/hydrochlorothiazide combination in elderly patients with mild-moderate hypertension. A double-blind, randomized, placebo-controlled study. Postgrad Med J 62:139-41, 1986

16. Veterans Administration Cooperative Study Group on Antihypertensive Agents: Low-dose captopril for the treatment of mild to moderate hypertension. Arch Intern Med 144:1947-53, Oct 84

17. Veterans Administration Cooperative Study Group on Antihypertensive Agents: Racial differences in response to low-dose captopril are abolished by the addition of hydrochlorothiazide. Br J Clin Pharmacol $14: 97 \mathrm{~S}-101 \mathrm{~S}, 1982$

Dr. Tuck is professor of medicine, University of California, Los Angeles, and chief, Endocrinology Section, Sepulveda Veterans Medical Center, Sepulveda, California.

Dr. Tuck, Endocrinology-111E, Sepulveda VAMC, 16111 Plummer Street, Sepulveda, California 91343. 